Compactness Theorems for Sequences of Riemannian Manifolds

\section{Christina Sormani}

Given a sequence of Riemannian manifolds, one may hope that there is a subsequence which converges to a possibly singular limit space. One may have sequences of spheres developing conical singularities (as in Figure 1), or thin deep wells (as in Figure 2), or perhaps even increasingly many wells (as in Figure 3). One may have a sequence of Riemannian manifolds which are not diffeomorphic to one another (as in Figure 4). Note that we consider sequences of distinct Riemannian manifolds that are not submanifolds of Euclidean space. In what sense might they converge? What are their limits?
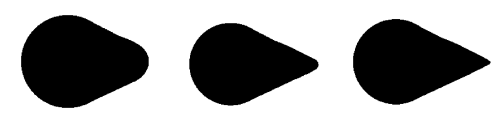

Figure 1. Developing a cone tip
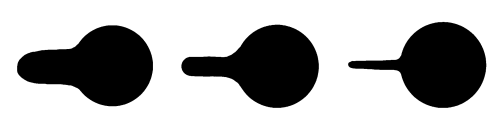

Figure 2. Thinner and thinner wells
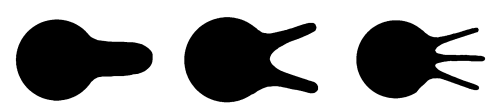

Figure 3. Increasingly many wells
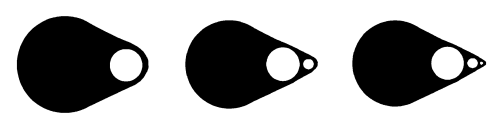

Figure 4. Increasing genus

In order to define a notion of convergence for distinct Riemannian manifolds, Gromov decided to view each Riemannian manifold, $\left(M_{i}, g_{i}\right)$, as a metric space, $\left(X_{i}, d_{i}\right)$. To define a distance between a pair of them, he embedded

Christina Sormani is a professor at Lehman College and the CUNY Graduate Center. She is currently a visitor at SCGP and IAS. Her email address is sormanic@gmai1.com.

For permission to reprint this article, please contact:

reprint-permission@ams .org

DOI: https://doi.org/10.1090/noti/1848 them into a common metric space, $Z$, via distance preserving maps, $\varphi_{i}: X_{i} \rightarrow Z$ :

$$
d_{Z}\left(\varphi_{i}(p), \varphi_{i}(q)\right)=d_{i}(p, q) \forall p, q \in X_{i} .
$$

He then took the Hausdorff distance, $d_{H}^{Z}$, between their images, $S_{i}=\varphi_{i}\left(X_{i}\right)$, as in Figure 5. Recall that $d_{H}^{Z}\left(S_{1}, S_{2}\right)$ is the smallest radius $r$ such that

$$
\forall z_{i} \in S_{i} \exists z_{j} \in S_{j} \text { such that } d_{Z}\left(z_{i}, z_{j}\right) \leq r .
$$

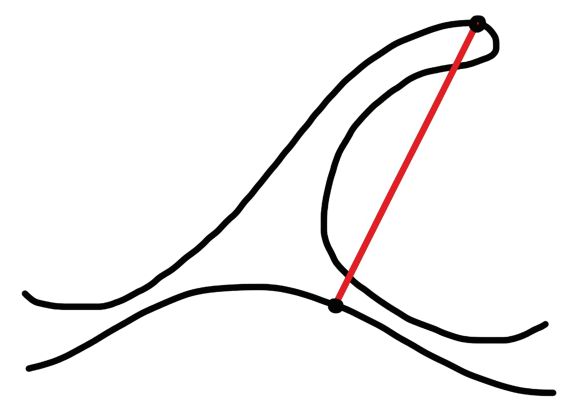

Figure 5. The Hausdorff distance between the black curves is the length of the red line.

More precisely the Gromov-Hausdorff distance between two metric spaces, $M_{i}=\left(X_{i}, d_{i}\right)$, is

$$
d_{G H}\left(M_{1}, M_{2}\right)=\inf \left\{d_{H}^{Z}\left(\varphi_{1}\left(X_{1}\right), \varphi_{2}\left(X_{2}\right)\right)\right\}
$$

where the infimum is over all compact metric spaces $Z$ and all distance preserving maps, $\varphi_{i}: X_{i} \rightarrow Z$. Gromov proved that $d_{G H}\left(M_{1}, M_{2}\right)=0$ iff $M_{1}$ and $M_{2}$ are isometric.

The sequence in Figure 1 converges in the GH sense to a sphere with a conical singularity. The sequence in Figure 2 converges to a sphere with a line segment attached to it. In general GH limits are metric spaces with geodesics, but they have no smooth structure.

Gromov's compactness theorem states that any sequence of metric spaces which have a uniform upper bound on diameter, $D$, and a uniform maximal number, $N(r)$, of disjoint balls of radius $r$ has a subsequence which converges in the GH sense. The sequence of manifolds with increasingly many wells in Figure 3 fails the hypothesis of Gromov's compactness theorem. The number of balls centered at the tips is increasing to infinity. In fact it has no subsequence converging in the GH sense. Ilmanen presented this example as a sequence that ought to converge to a sphere under some weak notion of convergence and asked what the notion could be.

In joint work with Wenger, we solved Ilmanen's question by introducing the intrinsic flat $(\mathcal{F})$ convergence. We viewed the oriented Riemannian manifolds as integral current spaces: metric spaces with oriented weighted biLipschitz charts, $M_{i}=\left(X_{i}, d_{i}, T_{i}\right)$. We defined the intrinsic 
flat distance between two integral current spaces to be:

$$
d_{\mathcal{F}}\left(M_{1}, M_{2}\right)=\inf \left\{d_{F}^{Z}\left(\varphi_{1 \#}\left(T_{1}\right), \varphi_{2 \#}\left(T_{2}\right)\right)\right\}
$$

where the infimum is over all complete metric spaces $Z$ and all distance preserving maps, $\varphi_{i}: X_{i} \rightarrow Z$. Yet instead of taking the Hausdorff distance between the images we took the flat distance, $d_{F}^{Z}$, between them.

The flat distance was first defined by Whitney and Federer-Fleming on $Z=\mathbb{E}^{N}$ as the infimum of the weighted volumes:

$$
d_{F}^{Z}\left(S_{1}, S_{2}\right)=\inf \left\{M(A)+M(B): A+\partial B=S_{1}-S_{2}\right\}
$$

where the infimum is over all generalized submanifolds, $A^{m}$ and $B^{m+1}$ as in Figure 6:

$$
\int_{S_{1}} \omega-\int_{S_{2}} \omega=\int_{\partial B} \omega+\int_{A} \omega=\int_{B} d \omega+\int_{A} \omega .
$$

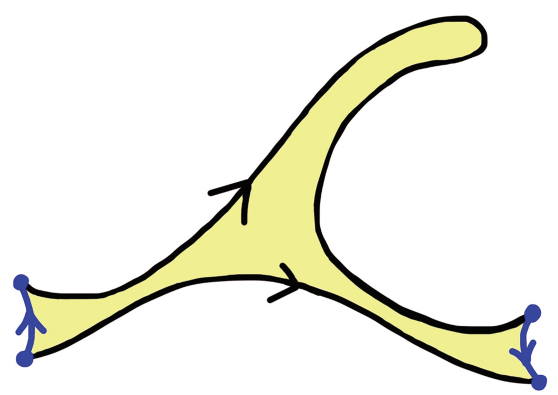

Figure 6. The flat distance between the black curves is the area of the yellow region plus the sum of the lengths of the blue curves.

We applied work of Ambrosio-Kirchheim to make this rigorous and prove that $d_{\mathcal{F}}\left(M_{1}, M_{2}\right)=0$ iff there is an orientation preserving isometry from $M_{1}$ to $M_{2}$.

Intuitively the $\mathcal{F}$ distance measures the filling volume between the Riemannian manifolds. The sequence in Figure 3 converges in the $\mathcal{F}$ sense to a standard sphere exactly as Ilmanen required. More generally, Wenger and I proved the limit spaces are metric spaces covered almost everywhere by biLipschitz charts that define an orientation and a weight (so they are rectifiable). We proved that whenever a sequence of manifolds has a GH limit, and a uniform upper bound on volume, then it has an $\mathcal{F}$ limit which is a subset of the GH limit. For example, the $\mathcal{F}$ limit of the sequence depicted in Figure 2 is only the sphere while the GH limit also has a line segment. See Figure 7.

Wenger's compactness theorem states that a sequence of oriented Riemannian manifolds with a uniform upper bound on volume and diameter has a subsequence converging in the $\mathcal{F}$ sense. However it is possible that the limit space is just the $\mathbf{0}$ space. This happens for example if the sequence of manifolds has volume converging to 0 .

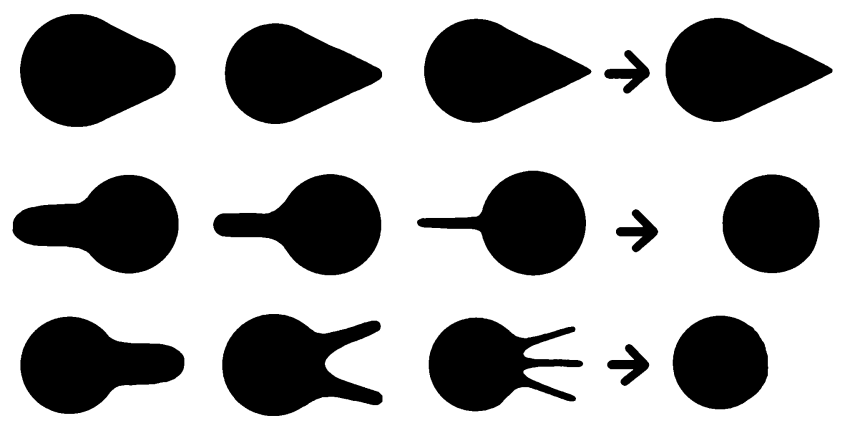

Figure 7. The intrinsic flat limits of our sequences.

Ideally one can show the $\mathrm{GH}$ and $\mathcal{F}$ limits agree, thus proving the $\mathrm{GH}$ limit is rectifiable and the $\mathcal{F}$ limit is not 0. For example the sequence in Figure 4 converges in the $\mathrm{GH}$ and $\mathcal{F}$ sense to the same rectifiable limit space with infinitely many holes.

The Tetrahedral Compactness Theorem states that if a sequence of manifolds satisfies a uniform noncollapsing condition on tetrahedra lying in the spaces, then a subsequence converges in the $\mathrm{GH}$ and $\mathcal{F}$ sense to the same rectifiable limit space. This theorem was proven jointly with Portegies, and improved by Nuñez-Zimbrón and Perales. This theorem and many more examples will be presented in the lecture.

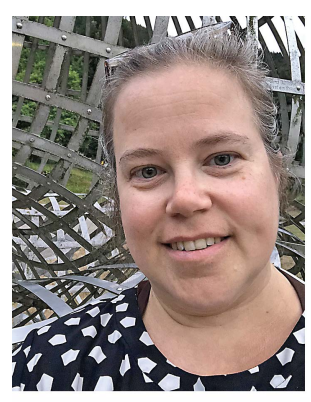

Christina Sormani

Credits

All images are courtesy of the author. 